\title{
Increased serum level of Nup88 protein is associated with the development of colorectal cancer
}

\author{
Zeng-Ren Zhao, Li-Jing Zhang, Yuan-Yuan Wang, Fang Li, \\ Ming-Wei Wang and Xiao-Feng Sun
}

\section{Linköping University Post Print}

N.B.: When citing this work, cite the original article.

The original publication is available at www.springerlink.com:

Zeng-Ren Zhao, Li-Jing Zhang, Yuan-Yuan Wang, Fang Li, Ming-Wei Wang and Xiao-Feng Sun, Increased serum level of Nup88 protein is associated with the development of colorectal cancer, 2012, Medical Oncology, (29), 3, 1789-1795.

http://dx.doi.org/10.1007/s12032-011-0047-1

Copyright: Humana Press (Springer Imprint) http://www.springer.com/

Postprint available at: Linköping University Electronic Press http://urn.kb.se/resolve?urn=urn:nbn:se:liu:diva-82065 
Increased serum level of Nup88 protein is associated with the development of colorectal cancer 


\section{Abstract}

Nucleoporin88 (Nup88) has been shown to be overexpressed in a wide variety of malignancies including colorectal cancer (CRC). However, no study about serum Nup88 in human CRC was reported. Therefore, in this study, we investigated the level of serum Nup88 protein and its relationships with clinicopathological variables in CRC. The serum concentration of Nup88 protein was determined by a quantitative sandwich enzyme-linked immunosorbent assay (ELISA) in 118 pre-operative serum samples, 66 post-operative and 96 healthy controls. Among the patients, the levels of CEA $(n=91)$ and CA19-9 $(n=87)$ in the pre-operative serum were measured, and DNA sequencing was performed in 12 CRCs and 2 samples from non-cancerous colon tissue. In the same patients, the level of pre-operative serum Nup88 was significantly higher than the post-operative Nup88 $(P=0.021)$. Furthermore, the level of pre-operative Nup88 was positively related to the depth of tumor invasion $(P=0.002)$ and advanced stage $(P=0.001)$. The level of pre-operative Nup88 in the left colon tended to be higher than that in the right colon and the rectum $(P=0.063)$. DNA sequencing results showed that there were two single nucleotide polymorphism, distributed in exon 6 (NM_002532.3:c.1044G>A $($ ACG - ACA, Thr $\rightarrow$ Thr $)$ and exon $10($ NM_002532.3:c.1389A>T, CCA - CCT, Pro $\rightarrow$ Pro $)$. Serum Nup88 might be a candidate for a new biomarker implicated in the development and aggressiveness of

CRCs. 


\section{Keywords}

Nup88, serum, colorectal cancer 


\section{Introduction}

Colorectal cancer (CRC), one of the most aggressive carcinomas, is the second leading cause of cancer mortality in the western world. Many Asian countries including China have experienced an increase of 2-4 times in the incidence of CRC during the past few decades [1], and about 40-50\% of patients may still die of local or distant recurrence after the surgery. It is therefore urgent to identify effective markers for the early diagnosis and treatment of CRC.

Nucleoporin88 (Nup88), one of the proteins termed "nucleoporins" (Nups), is localized at 17p13 and participates in the construction of nuclear pore complexes (NPCs). NPCs are embedded in the double-membraned nuclear envelope (NE) and mediate bidirectional nucleocytoplasmic transport [2]. Accordingly, Nup88 protein is involved in nuclear-cytoplasmic transport and cell growth. Nup88 has been shown in previous studies to be overexpressed in a wide variety of malignancies including gastroenteric carcinomas [3-5]. In our previous studies on tissues from CRC patients, Nup88 protein determined by immunohistochemistry was overexpressed in the primary tumor and lymph node metastasis of CRC compared to the normal colorectal mucosa, and the strong expression of Nup88 predicted a worse outcome in the patients [6-7]. The level of Nup88 was highly correlated with the aggressiveness of breast cancer [8]. A recent study has shown that Nup88 was localized on the spindles together with Nup214 during mitosis and its proper expression was critical for preventing aneuploidy formation and tumorigenesis [9]. Nup88 has been proposed as a tumor marker [10].

Up to now, there is no information on the clinical significance of serum Nup88 in CRC patients before and after surgery in comparison with healthy controls. In the present study, we collected the preand post-operative serum samples from patients with CRC as well as from healthy controls in order to investigate the association between the patients and controls, especially the levels of serum Nup88 
before and after surgery, and the relationships of the Nup88 with clinicopathological variables. 


\section{Materials and Methods}

\subsection{Patients and healthy controls}

One hundred eighteen pre-operative serum samples of CRC were collected and all these patients were histologically verified and treated at First Hospital of Hebei Medical University (Shijiazhuang, China) between 2009 and 2010. No patients had been prescribed chemotherapy or radiotherapy prior to surgery, and there was no evidence of any other malignancy. The post-operative serum was collected from 66 of the 118 patients and among of them, there are 58 matched (i.e., the 58 patients had the both pre and post-operative serum). Pre-operative serum samples were drawn within 7 days prior to surgery and the post-operative serum were collected after 7 days of surgery. The serum samples were collected during the hospital stay. The median age of CRC patients was 61 years (range from 36-86 years), and the male account to $66 \%$, and the female $34 \%$. The information of the tumor was based on the clinical and pathological examinations of post-operation; 25 tumors were located in the left colon, 24 in the right colon, and 55 in the rectum, 103 were non-mucinous adenocarcinomas and 15 mucinous/signet-ring cell carcinomas. The depth of tumor invasion was as follows: 9 involving muscularis, 15 with serosa and 72 with adventitia or over, A total of 73 patients were diagnosed with lymph node metastasis and 11 patients with distant metastasis.

For the healthy controls, serum samples were collected from 96 healthy volunteers (50 males and 46 females) without history of serological abnormalities, and the mean age was 58 years (range from 40-78 years, median age 58 years). Neither the age nor the sex was significantly different with the CRC patients.

The study was approved by the Institutional Ethical Committee of the First Hospital of Hebei Medical University, and informed consent was signed from all the participants in the study.

\subsection{Methods}

\section{ELISA}

Peripheral venous blood samples were collected, centrifuged at 2000g for 10 minutes and stored at $-70^{\circ} \mathrm{C}$ until used. The serum Nup 88 concentrations were determined by a quantitative sandwich enzyme-linked immunosorbent assay (ELISA, Human Nup88 ELISA kit (GBD, San Diego, CA). A standard curve was prepared using recombinant human Nup88 according to the manufacture's instructions. Each ELISA assay measured $100 \mu 1$ serum based on the manufacturer's directions. All analyses and calibrations were carried out in duplicate. Optical density was determined using a 
microplate reader (BIO-RAD) at wave length of 450nm for Nup88 determination. The concentrations were reported as $\mathrm{ng} / \mathrm{mL}$ Serum Nup88 concentrations were determined without knowledge of the main clinicopathological features of the patients studied.

In the present study, the same serum samples were used to quantify the serum levels of carcinoembryonic antigen (CEA) and carbohydrate antigen 19-9(CA19-9). The serum levels of CEA ( $\mathrm{n}=91)$ and CA19-9 ( $\mathrm{n}=87)$, were measured using commercial kits used clinically in our hospital, according to the manufacture's recommended protocol. CEA and CA19-9 were quantitated with the recommended cut-off values of $5 \mathrm{ng} / \mathrm{mL}$ and $37 \mathrm{U} / \mathrm{mL}$, respectively.

\section{Nucleotide sequence analysis}

Nup88 gene sequence was analyzed by extracting genomic DNA from the fresh CRC $(n=12)$ and non-cancerous colon tissues $(\mathrm{n}=2)$ with a tissue Gen DNA kit (CWBIO, China) according to its manufacturer's instructions. All of the coding nucleotide of the Nup88 gene was then directly PCR-amplified in a thermal cycler. The sequences of primers used for Nup88 analysis were listed in Table 1. PCR conditions were as follows: 1 cycle at $95^{\circ} \mathrm{C}$ for $4 \mathrm{~min}, 35$ cycles at $95^{\circ} \mathrm{C}$ for $40 \mathrm{sec}$, respective annealing temperature for $40 \mathrm{sec}$, and at $72^{\circ} \mathrm{C}$ for $40 \mathrm{sec}$, followed by 1 cycle at $72^{\circ} \mathrm{C}$ for 10 min. After confirmed by agarose electrophoresis and ethidium bromide staining, the PCR products were purified and automatically sequenced with an ABI PRISM 3730 (Applied Biosystems, California, USA), then analyzed with Chromas software version 2.0 (Gene Codes Corporation, Ann Arbor, MI ). All sequencing reactions were performed in both forward and reverse directions, by independent PCR.

\subsection{Statistical analysis}

The difference of Nup88 serum levels among pre-, post-operative and healthy controls was tested by Kmskal-Wallis test. Nup88 serum distributions in the paired pre-and post- operative CRC patients were compared using the paired-sample T test. Associations between pre-operative serum levels of Nup88 and clinicopathological variables were analyzed by Wilcoxon test or Kmskal-Wallis test. Differences with $P<0.05$ were considered to be statistically significant. 


\section{Results}

\section{Serum Nup88 in CRC patients and healthy controls}

The serum was isolated, including 118 cases of pre-operative serum, 66 cases of post-operative serum with CRC, and 96 healthy controls, and measured by the ELISA assay. The levels of Nup88 ranged from 0.25 to $69.08 \mathrm{ng} / \mathrm{mL}$ in the pre-operative serum of CRC patients, 0.21 to $13.51 \mathrm{ng} / \mathrm{mL}$ in the 66 cases of post-operative serum, and 0.73 to $9.87 \mathrm{ng} / \mathrm{mL}$ in the 96 healthy controls, respectively (Fig.1).

The levels of nup88 in the pre-operative serum had a trend of increase compared with either the post-operative $(P=0.089)$ or the control $(P=0.091)$. There was no significant difference between the post-operative and the controls $(P=0.856)$.

We further compared the serum Nup88 level of pre- with post- operative serum in the matched 58 cases of CRC (i.e., the same patients), the levels of Nup88 in the post-operative serum were notably decreased compared with the pre-operation $(3.14 \pm 1.97$ vs $6.19 \pm 10.99, P=0.021$, Fig.2).

\section{Relationship between Nup88 levels in the pre-operative serum and clinicopathological variables}

We analyzed the relationships between pre-operative serum Nup88 levels (defined as the median and interquartile range) and the clinicopathological variables (Table 2). The level of serum Nup88 was different among right colon, left colon and the rectum $\left(\chi^{2}=5.517, P=0.063\right)$. The further comparison showed that the Nup88 in the left colon was significantly higher than in the rectum $(P=0.023)$, however, there was no difference of right colon with either left colon $(P=0.197)$ or the rectum $(P=0.314)$.

Serum Nup88 levels were related to the depth of tumor invasion. Along with the tumor penetrated to the fat layer, Nup88 level was remarkably increased ( $\left.\chi^{2}=12.092, P=0.002\right)$, whereas there was no significant difference when infiltrated to the muscular layer and the serosa $(P=0.20)$. Nup88 
levels of respective TNM stage (I-IV)were shown in Table 2. The patients with advanced stage (III \pm IV) had a significantly higher level of Nup88 than those with the earlier stage ( $I \pm I I),(P=0.001)$. There was no significant difference between stage I and II $(P=0.767)$, neither did in stage III and IV $(P=0.893)$. Serum Nup88 level in mucinous/signet-ring cell carcinomas had a trend of increase compared with that in non-mucinous carcinomas $(P=0.088)$. The level of serum Nup88 was higher in CEA positive group than that in CEA negative, although the difference was not statistically significant $(P=0.116)$. No significant difference in Nup88 levels was found among gender, age, tumor size, lymph node status, distant metastasis, accompanied adenoma/polypus or not and CA19-9 expression $(P>0.05$, Table 2).

\section{Relationship between Nup88 levels in the post-operative serum and clinicopathological variables}

We examined the relationship between post-operative serum Nup88 and the clinicopathological variables and did not show any significant correlations, including patient's gender, age, tumor location, tumor size, invasion depth, lymph node status, TNM stage, histological type, CEA and CA19-9 expression $(P>0.05$, data not shown).

\section{DNA sequencing results of Nup88}

To examine whether there is any mutation in the Nup88 gene (17p13), we sequenced all of the coding nucleotides (2332 base pairs, nt 27 to 323 , nt 2995 to 3164 , nt 5543 to 5668 , nt 8888 to 8974 , nt 10768 to 10944 , nt 14434 to 14620 , nt 15431 to 15578 , nt 20027 to 20125 , nt 24696 to 24786 , nt 28015 to 28116 , nt 30717 to 30875 , nt 31773 to 31898 , nt 32007 to 32072 , nt 32211 to 32291 , nt 32567 to 32693 , nt 32851 to 32969 , nt 33408 to 33471 ) that are distributed to 17 exons. The results showed two 
single nucleotide polymorphisms, distributed in exon 6 (NM_002532.3:c.1044G>A (ACG - ACA, Thr

$\rightarrow$ Thr) and exon 10 (NM_002532.3:c.1389A>T, CCA - CCT, Pro $\rightarrow$ Pro) respectively (Fig. 3). 


\section{Discussion}

In the present study,. We found that the CRC patients before the operation had a relative higher level of serum Nup88 than the healthy controls, whereas, there was no significant difference between the post-operative and the healthy controls. So we presumed that increased level of Nup88 in the pre-operative serum might be of some value for the clinical diagnosis of CRC.

We further compared the levels of Nup88 in the patients before and after operation ( 7 days after the surgery), after the operation, Nup88 was notably decreased, especially in the matched patients. It is interesting to observe the post-operative serum Nup88 level in different time points afer surgery. Unfortunately we do not have follow up data, otherwise it is more important to see whether the level of Nup88 changed as the patients had local or distant recurrences. If so we could consider to use Nup88 for predicting the patients' outcome.

We also analyzed the relationships of pre-operative serum Nup88 with the clinicopathological variables, and found that the Nup88 levels were related to the depth of tumor invasion and serum Nup88 level in mucinous/signet-ring cell carcinomas had a trend of increase compared with that in non-mucinous carcinomas $(P=0.088)$. Along with the tumor penetrated to the fat layer, the Nup88 was remarkably increased. It has been reported that in endometrial carcinoma, growing levels of Nup88 mRNA expression was correlated with the depth of myometrial invasion [4]. In our study, the levels of Nup88 in the pre-operative serum of CRC patients with advanced stage (III \pm IV) were much higher than the earlier stage ( $I \pm$ II ). In our previous study, Nup88 immunostaining was found to be positively related to the infiltrative growth and reversely to the grade of differentiation, and predicted a worse outcome in CRC patients with distal tumors [7]. Agudo D et al [11] also found that Nup88 mRNA overexpression was associated with high aggressiveness of breast cancer. It has been reported that Nup88 specifically binds the tail domain of lamin A [12], together with Nup214, involved in the 
mitosis and the proper expression of Nup88 is critical for preventing aneuploidy formation and tumorigenesis [10].

Furthermore, we also found the level of serum Nup88 in the left colon tend to be higher than that in the right colon and the rectum $(P=0.063)$. A lot of investigations have found left-sided colonic tumor location and mucinous/signet-ring cell carcinomas were significantly correlated with poor overall survival [13-14]. We also investigated the relationship between post-operative serum Nup88 and the clinicopathological features and did't find any correlation. To sum up, we speculate increased preoperative serum levels of Nup88 are associated with the tumorigenesis of CRC.

We finally sequenced the all of the coding nucleotides (2332 base pairs) of Nup 88 gene and found two single nucleotide polymorphism (NM_002532.3:c.1044G>A and (NM_002532.3:c.1389A>T), respectively. A recent genetic screen identified a partial loss-of-function mutation in Arabidopsis MOS7/Nup88 that causes defects in basal immunity, Resistance (R) protein-mediated defense and systemic acquired resistance. In Drosophila and mammalian cells, exportin-mediated nuclear export of activated Rel/NFkB transcription factors is enhanced in Nup88 mutants resulting in immune response failure [15].

\section{Conclusion}

In the present study, the level of pre-operative serum Nup88 had a trend of increase compared with the healthy controls, and after the operation, the level of Nup88 was significantly decreased. Furthermore, the level of Nup88 was related to the advanced stage. We supposed that serum Nup88 might be a candidate for a new marker and implicated in the tumorigenesis and development of CRC. 


\section{Conflict of interest statement}

We declare that there is no conflict of interest that would prejudice the impartiality of this scientific work. 


\section{Ackowledgment}

This study was supported by the National Natural Science Foundation of China (No.30772115). 


\section{References}

1. Sung JJ, Lau JY, Goh KL, Leung WK: Increasing incidence of colorectal cancer in Asia: implications for screening. Lancet Oncol 2005; 6: 871-876.

2. Fahrenkrog B, Aebi U. The nuclear pore complex:nucleocytoplasmic transport and beyond. Nat. Rev. Mol. Cell. Biol. 2003; 4:757-766

3. Zhang H, Schneider J, Rosdahl I. Expression of p16, p27, p53, p73 and Nup88 proteins in matched primary and metastatic melanoma cells. Int J Oncol 2002; 21: 43- 48.

4. Schneider J, Martínez-Arribas F, Torrejón R. Nup88 expression is associated with myometrial invasion in endometrial carcinoma. Int J Gynecol Cancer. 2010 ; 20(5):804-808.

5. Brustmann H, Hager M. Nucleoporin 88 expression in normal and neoplastic squamous epithelia of the uterine cervix. Ann Diagn Pathol. 2009; 13(5):303-307.

6. Zhang ZY, et al. Nup88 expression in normal mucosa, adenoma, primary adenocarcinoma and lymph node metastasis in the colorectum. Tumour Biol. 2007; 28(2):93-99.

7. Emterling A, et al.Clinicopathological significance of Nup88 expression in patients with colorectal cancer. Oncology. 2003; 64(4):361-369.

8. Agudo D, et al. Nup88 mRNA overexpression is associated with high aggressiveness of breast cancer. Int J Cancer. 2004 ;109(5):717-720.

9. Xu S, Powers MA: Nuclear pore proteins and cancer. Semin Cell Dev Biol 2009; 20:620-630.

10. Hashizume C, Nakano H, Yoshida K, Wong RW. Characterization of the role of the tumor marker Nup88 in mitosis. Mol Cancer. 2010; 24;9:119.

11. Agudo D, et al. Nup88 mRNA overexpression is associated with high aggressiveness of breast cancer. Int J Cancer, 2004, 109(5): 717-720.

12. Lussi YC, Hügi I, Laurell E, Kutay U, Fahrenkrog B. The nucleoporin Nup88 is interacting with 
nuclear lamin A. Mol Biol Cell. 2011; 22(7):1080-1090.

13. Maksimović S. Survival rates of patients with mucinous adenocarcinoma of the colorectum, Med Arh. 61(2007), 26-29.

14. Kanemitsu Y, et al. Survival after curative resection for mucinous adenocarcinoma of the colorectum, Dis Colon Rectum. 46(2003), 160-167.

15. Wiermer M, et al. Nucleoporin MOS7/Nup88 contributes to plant immunity and nuclear accumulation of defense regulators. Nucleus. 2010 ;1(4):332-333. 
Table 1 Primers used for Nup88 DNA sequencing

\begin{tabular}{|c|c|c|c|}
\hline Nup88 & & Pimer sequence (5'-3') & Product \\
\hline \multirow{2}{*}{ CDS1 } & forward & CTTGGGCTTCAGAAATAATGGT & \multirow[b]{2}{*}{$750 \mathrm{bp}$} \\
\hline & reverse & CCAGAGCACTCATCCGTCACTA & \\
\hline \multirow{2}{*}{ CDS2 } & forward & TGTGAGTTAATGGTACAAGTGTGA & \multirow{2}{*}{$519 \mathrm{bp}$} \\
\hline & reverse & CAACGTGGTACTTCAGGCAGA & \\
\hline \multirow{2}{*}{ CDS3 } & forward & AGAATCACTTGAACCTGGGAG & \multirow{2}{*}{$480 \mathrm{bp}$} \\
\hline & reverse & AAGGAATAGTGGTAAGTTAGAGCAG & \\
\hline \multirow{2}{*}{ CDS4 } & forward & CAATAGTGAGATGACTGGACAAGG & \multirow{2}{*}{$559 \mathrm{bp}$} \\
\hline & reverse & ACGACGAATCCCTGTTCTACTA & \\
\hline \multirow{2}{*}{ CDS5 } & forward & TCTTTTCAGTCCATTGCCTACA & \multirow{2}{*}{$484 b p$} \\
\hline & reverse & GCCAAATGACCTGTACTAACTCTT & \\
\hline \multirow{2}{*}{ CDS6 } & forward & TCTGGTTGAGAAACACAGAGTG & \multirow{2}{*}{$560 \mathrm{bp}$} \\
\hline & reverse & GCAАССТTCTAАСТTCTTTCСАСТ & \\
\hline \multirow{2}{*}{ CDS7 } & forward & GACAGGGTTTCAGTATGTTGGC & \multirow{2}{*}{$562 \mathrm{bp}$} \\
\hline & reverse & GTGATCTGGACCAAAGACCACT & \\
\hline \multirow{2}{*}{ CDS8 } & forward & TGGAACCAAACCCACAGTATC & \multirow{2}{*}{$658 \mathrm{bp}$} \\
\hline & reverse & TATCCCAGACCCTGATTGAGT & \\
\hline \multirow{2}{*}{ CDS9 } & forward & GAAAGCGTATGCCTATGTCAGA & \multirow{2}{*}{$520 \mathrm{bp}$} \\
\hline & reverse & CAGAAAGAAGGGTAGGAGTTAGC & \\
\hline \multirow{2}{*}{ CDS10 } & forward & CTACAGTTAGGGATTCCTGAGTGA & \multirow{2}{*}{$691 b p$} \\
\hline & reverse & TGATGATTCCAGGAGGCAGT & \\
\hline \multirow{2}{*}{ CDS11 } & forward & GCATTGGAATAATAAGGGTGTAGC & \multirow{2}{*}{$535 \mathrm{bp}$} \\
\hline & reverse & TGTTTATCATTCTCAGGTGCCA & \\
\hline \multirow{2}{*}{ CDS12-14 } & forward & TGACAGAGCGAGACTGCATC & \multirow{2}{*}{$825 b p$} \\
\hline & reverse & CATAGGAAGGGTTAGGGGAGA & \\
\hline \multirow{2}{*}{ CDS15-16 } & forward & CATACCTACGGAACTGAACTGAT & \multirow{2}{*}{$916 \mathrm{bp}$} \\
\hline & reverse & AGGTTTCAGGTGGATAGCAAT & \\
\hline \multirow{2}{*}{ CDS17 } & forward & TGACTATGGTGGGAGGATTGA & \multirow{2}{*}{$410 \mathrm{bp}$} \\
\hline & reverse & TTACAATATGGGTTTAAGCCTTC & \\
\hline
\end{tabular}


Table 2 Relationship between Nup88 levels in the pre-operation serum and clinicopathological variables

\begin{tabular}{|c|c|c|c|c|}
\hline Variables & $\mathbf{n}$ & Nup88 $^{a}$ & Statistic value & $P$-value \\
\hline Gender & $\mathrm{n}=104$ & & -0.103 & 0.918 \\
\hline Male & 69 & $3.041(1.675 \sim 4.504)$ & & \\
\hline Female & 35 & $2.507(1.5 \sim 4.451)$ & & \\
\hline Age & $\mathrm{n}=104$ & & -0.407 & 0.684 \\
\hline$<60$ & 45 & $2.709(1.468 \sim 4.650)$ & & \\
\hline$\geqslant 60$ & 59 & $3.019(1.703 \sim 4.318)$ & & \\
\hline Tumor location & $\mathrm{n}=104$ & & 5.517 & 0.063 \\
\hline Left colon & 25 & $3.522(2.286 \sim 5.156)$ & & \\
\hline Right colon & 24 & $2.954(1.782 \sim 4.491)$ & & \\
\hline Rectum & 55 & $2.563(1.140 \sim 4.101)$ & & \\
\hline Tumor size & $\mathrm{n}=84$ & & -0.110 & 0.912 \\
\hline$<40 \mathrm{~cm}^{3}$ & 33 & $3.064(2.199 \sim 4.491)$ & & \\
\hline$\geqslant 40 \mathrm{~cm}^{3}$ & 51 & $3.327(1.678 \sim 4.718)$ & & \\
\hline Depth of invasion & $\mathrm{n}=96$ & & 12.092 & $0.002 *$ \\
\hline Muscular layer & 9 & $2.132(1.445 \sim 3.374)$ & & \\
\hline Serosa & 15 & $1.123(0.441 \sim 2.709)$ & & \\
\hline Fat layer & 72 & $3.367(2.358 \sim 4.684)$ & & \\
\hline Lymph node metastasis & $\mathrm{n}=118$ & & 0.891 & 0.373 \\
\hline No & 45 & $2.619(1.321 \sim 3.645)$ & & \\
\hline Yes & 73 & $2.643(1.422 \sim 4.346)$ & & \\
\hline Distant metastasis & $\mathrm{n}=100$ & & -1.361 & 0.174 \\
\hline No & 89 & $2.943(1.663 \sim 4.442)$ & & \\
\hline Yes & 11 & $3.407(2.889 \sim 5.302)$ & & \\
\hline TNM stage & $\mathrm{n}=99$ & & 10.871 & $0.012 *$ \\
\hline I & 7 & $2.132(1.548 \sim 3.370)$ & & \\
\hline II & 36 & $2.591(1.22 \sim 4.214)$ & & \\
\hline III & 45 & $3.706(2.308 \sim 5.561)$ & & \\
\hline IV & 11 & $3.407(2.889 \sim 5.302)$ & & \\
\hline Histological type & $\mathrm{n}=118$ & & -1.709 & 0.088 \\
\hline Non-mucinous & 103 & $2.563(1.378 \sim 4.258)$ & & \\
\hline $\begin{array}{l}\text { Mucinous/signet-ring } \\
\text { cell carcinoma }\end{array}$ & 15 & $3.66(2.35 \sim 4.45)$ & & \\
\hline Accompanied adenoma & $\mathrm{n}=118$ & & -1.355 & 0.176 \\
\hline No & 106 & $2.709(1.514 \sim 4.431)$ & & \\
\hline Yes & 12 & $2.258(0.988 \sim 3.335)$ & & \\
\hline CEA & $\mathrm{n}=91$ & & -1.570 & 0.116 \\
\hline Negative & 48 & $2.672(1.18 \sim 4.423)$ & & \\
\hline Positive & 43 & $3.25(2.342 \sim 4.913)$ & & \\
\hline CA19-9 & $\mathrm{n}=87$ & & -0.893 & 0.372 \\
\hline Negative & 67 & $2.725(1.678 \sim 4.49)$ & & \\
\hline Positive & 20 & $3.645(1.903 \sim 4.923)$ & & \\
\hline
\end{tabular}

${ }^{\mathrm{a}}$ The level of Nup88 with the median and interquartile range

$* P<0.05$ 


\section{Figure legends}

Fig. 1. The levels of serum Nup88 protein in the pre- and post-operative patients with CRC, and healthy controls. Serum Nup88 level was defined as mean $\pm 95 \%$ CI. the level of serum Nup88 in the pre-operative patients had a trend of increase than the post-operative $(P=0.089)$ and healthy controls $(P=0.091)$.

Fig. 2. The levels of pre- and post- operative serum Nup88 protein in the matched cases of CRC (defined as mean $\pm 95 \% \mathrm{CI}$ ). The level of Nup88 in the post-operative serum was notably decreased than in the pre-operative serum $(P=0.021)$.

Fig. 3. DNA sequencing of Nup88 CDS. All of the coding nucleotides that are distributed to 17 exons was sequenced with an ABI PRISM 3730 automated DNA Analyser. And two single nucleotide polymorphism were found, distributed in exon 6 (NM_002532.3:c.1044G>A) and exon 10 (NM_002532.3:c.1389A>T) respectively. The changed nucleotide was labeled with red. 